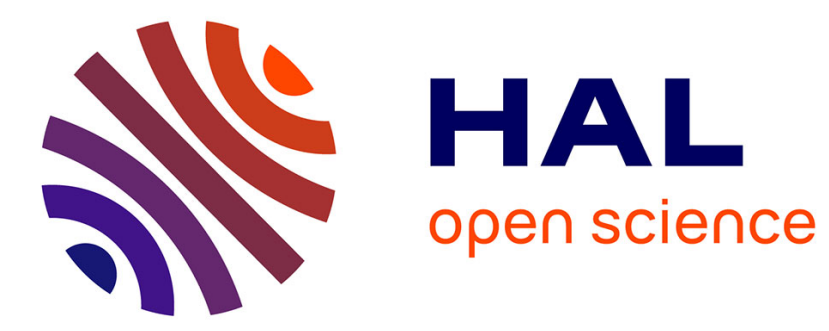

\title{
Créer un climat de confiance dans un cours de français sur objectifs universitaires sur Moodle
}

Chae-Yeon Bournel-Bosson, Jovan Kostov

\section{To cite this version:}

Chae-Yeon Bournel-Bosson, Jovan Kostov. Créer un climat de confiance dans un cours de français sur objectifs universitaires sur Moodle. Recherche et pratiques pédagogiques en langues de spécialité - Cahiers de l'APLIUT, 2017, Confiance, reliance et apprentissage des langues dans l'enseignement supérieur, 37 (1), 10.4000/apliut.5908 . halshs-01705970

\section{HAL Id: halshs-01705970 \\ https://shs.hal.science/halshs-01705970}

Submitted on 15 May 2020

HAL is a multi-disciplinary open access archive for the deposit and dissemination of scientific research documents, whether they are published or not. The documents may come from teaching and research institutions in France or abroad, or from public or private research centers.
L'archive ouverte pluridisciplinaire HAL, est destinée au dépôt et à la diffusion de documents scientifiques de niveau recherche, publiés ou non, émanant des établissements d'enseignement et de recherche français ou étrangers, des laboratoires publics ou privés.

$$
\text { Copyright }
$$



langues de spécialité

Cahiers de l'Apliut

Vol. $37 \mathrm{~N}^{\circ} 1$ | 2018

Confiance, reliance et apprentissage des langues dans l'enseignement supérieur

\section{Créer un climat de confiance dans un cours de français sur objectifs universitaires sur Moodle} Inspiring Self-Confidence in French for Academic Purposes through the Use of Moodle

Chae-Yeon Bournel-Bosson et Jovan Kostov

\section{OpenEdition Journals}

Édition électronique

URL : http://journals.openedition.org/apliut/5908

DOI : 10.4000/apliut.5908

ISSN : 2119-5242

Éditeur

APLIUT

\section{Référence électronique}

Chae-Yeon Bournel-Bosson et Jovan Kostov, « Créer un climat de confiance dans un cours de français sur objectifs universitaires sur Moodle», Recherche et pratiques pédagogiques en langues de spécialité [En ligne], Vol. $37 \mathrm{~N}^{\circ} 1$ | 2018, mis en ligne le 08 février 2018, consulté le 30 avril 2019. URL : http:// journals.openedition.org/apliut/5908; DOI : 10.4000/apliut.5908 


\title{
Créer un climat de confiance dans un cours de français sur objectifs universitaires sur Moodle
}

Inspiring Self-Confidence in French for Academic Purposes through the Use of Moodle

\author{
Chae-Yeon Bournel-Bosson et Jovan Kostov
}

\section{Introduction}

Depuis le lancement de la "stratégie numérique» en 2013 par le ministère de l'Enseignement supérieur et de la recherche, nous assistons à un recours croissant à l'utilisation du numérique pour développer les potentialités pédagogiques (AlbernheGiordan et Charnet). En parallèle, notre expérience d'enseignement a révélé une certaine réticence des étudiants à l'utilisation des environnements numériques de travail (ENT), qui serait notamment due à un changement dans leur culture d'enseignement / apprentissage. Cela impliquerait une nécessité de prise en compte de la "diversité constitutive » des étudiants (Castellotti).

2 Il paraît alors essentiel de travailler sur la mise en confiance des étudiants vis-à-vis de l'enseignement à distance sur les plateformes d'apprentissage pour leur acceptation et, pour aller plus loin, leur satisfaction. En effet, ces dispositifs leur ouvrent de nouvelles perspectives quant à l'organisation $\mathrm{du}$ travail universitaire (autonomie, responsabilisation).

Dans cette contribution, nous allons exposer quatre actions immédiatement transposables dans le cadre d'un cours à distance et susceptibles d'améliorer la confiance des étudiants. Plus particulièrement, nous allons analyser les résultats obtenus de la mise en place d'un enseignement entièrement dispensé à distance intitulé «Parlons FOU ! (B2)». À travers des actions concrètes, nous voulons démontrer que la confiance est proportionnée non seulement à la maîtrise technique de la plateforme mais également à 
la capacité de l'enseignant à inciter le réemploi du savoir des étudiants et à instaurer un climat convivial au sein du cours.

\section{Quelques remarques sur le contexte}

\subsection{Le pôle d'élaboration de ressources linguistiques (Perl)}

Perl est un service partagé du consortium université Sorbonne Paris Cité (USPC). Sa mission consiste à développer des formations innovantes en langues (anglais, FLE, espagnol, allemand) grâce aux potentialités pédagogiques offertes par les technologies de l'information et de la communication (TIC), notamment par la plateforme Moodle. Le Perl fédère les ressources linguistiques sur une plateforme dédiée ${ }^{1}$ accessible par tous les étudiants des établissements membres de l'USPC. Les formations servent de support aux cours entièrement dispensés à distance, hybrides ou en présentiel enrichi, et nécessitent un tutorat à distance.

\subsection{La formation «Parlons FOU! (B2)»}

5 Dans le cadre du Perl, nous avons créé ${ }^{2}$ une formation de français sur objectifs universitaires (FOU) de niveau B2 entièrement dispensée à distance : Parlons FOU! (B2). Toutes les ressources mises en place au Perl sont expérimentées auprès des étudiants de l'USPC. Ces expérimentations nous permettent d'améliorer certains aspects pédagogiques et d'adapter les formations à un public plus large. Parlons FOU! (B2) a fait l'objet d'une expérimentation au premier semestre de l'année universitaire 2016-2017, auprès d'une dizaine d'étudiants en première année de licence de psychologie inscrits à l'université Paris-Diderot.

Ce cours fait désormais partie intégrante de l'offre de formation du centre de ressources en langues (CRL) de cette même université. Il s'adresse aux étudiants de toutes les disciplines universitaires de niveau $\mathrm{B} 2^{3}$ du Cadre européen commun de référence pour les langues (Conseil de l'Europe) en français. Il vise à diagnostiquer et à développer les compétences linguistiques nécessaires à certains usages académiques du français. Il se déroule intégralement en ligne et s'articule sur 12 séances réparties en trois modules : le module I a pour thème «Suivre un cours magistral » (4 séances), le module II s'intitule «Maîtriser les écrits universitaires » (5 séances), et le module III «Présenter un exposé oral " (3 séances), à raison de deux heures de travail par séance, soit 24 heures de formation dans le semestre. Dans le module I, orienté vers la compréhension orale, les étudiants apprennent la méthodologie d'un cours de type « cours magistral» (CM), de la prise de notes et la planification des révisions en vue des examens. Dans le module II, nous consacrons une attention particulière à la production écrite: les étudiants apprennent à construire une problématique, à faire des recherches, à rédiger un plan détaillé, une introduction, une conclusion et une bibliographie. Enfin, dans le module III, les étudiants sont amenés à développer leur expression orale à travers la rédaction des fiches mnémotechniques, le développement de l'art oratoire et l'élaboration des supports visuels.

Chaque séance débute par un questionnaire autoréflexif sur les habitudes de travail à l'université (cf. annexes 1 et 2) ou par une découverte culturelle selon chaque module. Ensuite, elle comporte des activités autocorrigées et se termine par une activité de 
production. Chaque module est sanctionné par un devoir final où les étudiants mobilisent les compétences précédemment acquises.

\section{Comment améliorer la confiance des étudiants ?}

\subsection{Développer la maîtrise technique}

Nous proposons à tous les étudiants une séance 0 intitulée «Je découvre Moodle! ». Son but est de les inciter à se familiariser avec la plateforme afin de participer pleinement à toutes les activités en ligne. Dans la plupart des cas, ils n'ont jamais suivi de cours sur Moodle. Cette plateforme est souvent utilisée comme une zone de dépôt des supports de cours, sans que ses potentialités techniques et pédagogiques soient réellement exploitées. Les étudiants apprennent à effectuer un test en ligne sur Moodle, à rendre un devoir, à échanger dans le forum, et à envoyer un message à travers la messagerie instantanée. Elle leur permet aussi de comprendre les différents types de parcours sur Moodle: l'enseignant peut proposer ou imposer un parcours en réglant le passage d'une activité à une autre. Cette séance est plutôt négligée par les étudiants qui parcourent Moodle de façon intuitive et qui comprennent assez rapidement son fonctionnement. Mais ceux qui n'ont pas parcouru cette séance de découverte se sont vite retrouvés en difficulté lors du passage d'un module à l'autre, car ils n'avaient pas compris le système de progression qui permet de s'autoévaluer en marquant une activité comme achevée et de passer aux activités suivantes. Lorsque l'étudiant sait pleinement user des potentialités de la plateforme, il est en position d'apprécier non seulement l'enseignement dispensé, mais aussi de se sentir valorisé et d'avoir l'impression que celui-ci est une nouveauté, ce que confirment les paroles d'un.e de nos étudiant.e.s :

«Ce que je plus aimé c'est [...] que cette plateforme ne suit la forme classique et ancienne d'enseignement. $»^{4}$ (Témoignage d'un.e étudiant.e)

\subsection{Réemployer les savoirs de l'étudiant}

Le fil directeur du cours est le thème du « kif artistique ». Le module I se termine par un devoir final qui consiste à comprendre une conférence de Florence Servan Schreiber (2012) intitulée «Le pouvoir de la gratitude» dans laquelle elle soulève la nécessité d'avoir des «kifs» dans la vie. À partir de ce moment, les étudiants travaillent sur une œuvre artistique qui leur plait tout au long des modules II et III. Le devoir final du module II consiste à rendre un développement structuré sur une œuvre artistique dont ils font émerger une problématique. À la fin du module III, les étudiants remettent un document enregistré en ligne grâce à PoodLL $5^{5}$ qui reprend leur introduction du module II et qu'ils réadaptent à l'oral, ainsi qu'un diaporama illustrant leur exposé. Cette tâche convient aux étudiants de n'importe quelle discipline: ils se sentent en confiance par rapport à l'œuvre qu'ils maîtrisent. Ils peuvent, ainsi, la faire connaître aux autres et l'approfondir. C'est ce qui ressort dans le commentaire d'un étudiant :

«Les activités du kif artistique ,mon fait beaucoup plaisir de les réaliser, parce que elle utilisent un thème que nous intéresse pour travailler avec la langue française,servant de stimulus. » (Témoignage d'un.e étudiant.e) 


\subsection{Créer et renforcer le lien social entre les étudiants}

10 Le risque d'isolement ou d'abandon dans le cadre d'un enseignement hybride ou entièrement dispensé à distance a été souligné dans de nombreuses études (Dussarps, Dussarps et Paquelin, Guillemet). Pour pallier ce problème, la plateforme Moodle dispose de plusieurs outils qui permettent à l'étudiant de s'exprimer ou de contacter ses pairs dans des espaces dédiés. Voici ce que nous avons mis en place.

11 Base de données. L'objectif de la première séance est de mieux connaître les étudiants qui participent au cours. Chacun remplit une fiche de présentation contenant son nom, son prénom, une photo, son cursus, ses loisirs et son projet d'études. Ils doivent également s'enregistrer via PoodLL pour révéler une information personnelle de leur choix qui n'est pas mentionnée sur la fiche. Les étudiants peuvent consulter les fiches de leurs pairs et les commenter pour dire ce qu'ils ont appris grâce à l'enregistrement déposé.

Chat. Cet espace est consacré à une communication synchrone pour les étudiants qui effectuent simultanément une séance. Cependant, nous constatons que cet espace n'est jamais utilisé. En nous interrogeant sur les facteurs de cet échec, nous avons émis les hypothèses que (1) les étudiants ne se connectent pas en même temps, (2) ils ne savent pas quoi se dire, (3) le travail à effectuer n'a pas un caractère collaboratif, ce qui exclut d'emblée son utilisation, (4) l'activité « Chat » n'est pas si simple à utiliser. De ce fait, nous avons désactivé cet outil lors du réajustement de la formation.

Forum de discussion. Très souvent, le forum de discussion est délaissé aussi bien de la part des enseignants que des étudiants. C'est le constat que l'on peut observer chez Guillemet ( op. cit.). Cela étant, nous avons voulu explorer les potentialités de cet espace d'échange dans le but de renforcer le lien social entre les étudiants. Le forum, que nous avons appelé «Pour le plaisir ! ", est une activité facultative. Les étudiants peuvent s'exprimer sur euxmêmes et sur les coutumes de leur pays d'origine, donner leur avis sur un sujet, etc. Livrer leurs opinions à la communauté du cours est un signe de la volonté d'aller vers autrui et permet de donner un visage plus humain au distanciel. Les étudiants ont participé au forum en moyenne une fois par semaine sur douze semaines.

Questionnaire. De premier abord, cet outil ne semble pas être le plus approprié pour la création d'un lien social entre les participants du cours. Toutefois, il offre des avantages de par son côté participatif et on peut l'exploiter très largement pour que les étudiants répondent librement à des questions ouvertes, en rapport avec les thèmes de la séance. À la fin, l'étudiant peut consulter l'ensemble des contributions pour constater la divergence ou la convergence de son avis avec ceux des autres participants. Tous les étudiants du cours ont répondu qu'ils ont consulté les réponses de leurs pairs, ce qui nous a conforté dans l'idée que cet outil pouvait en effet créer du lien.

Atelier. L'atelier a été utilisé pour la réalisation du devoir final du module II. Cela a été mis en place pour inciter les étudiants à faire un retour sur le travail d'un pair, ce qui peut permettre de prendre conscience des points à améliorer dans leurs propres travaux. L'outil a essentiellement servi de moyen d'entraide à la réussite du devoir final. Cependant, le fait d'évaluer un pair ne s'est pas toujours avéré facile. Nous avons constaté un blocage chez une étudiante qui s'est sentie mal à l'aise dans cet exercice d'évaluation des pairs car elle ne voulait pas être dans la position de pénaliser le devoir d'un autre de ses pairs. Cela nous incite à mieux expliquer aux étudiants que le travail via l'atelier sert seulement d'entraide et d'apport d'un regard extérieur et nouveau. Les outils mentionnés 
précédemment doivent jouer pleinement leur rôle pour que les étudiants soient corrigés par une personne à laquelle ils font confiance.

\subsection{Renforcer l'interaction entre l'enseignant-tuteur et les étudiants} outils les plus privilégiés dans notre cours sont la messagerie personnelle comprise dans Moodle, les forums de discussion et les feedbacks de l'activité Devoir - tous des moyens de communication asynchrones. Chaque étudiant a reçu trois messages du tuteur à trois moments différents chaque semaine: un message collectif indiquant l'ouverture de la séance via un forum des nouvelles, un message collectif indiquant la fermeture prochaine de la séance via le forum des nouvelles, et un message individuel donnant un feedback sur le travail fourni dans la semaine via la zone de feedbacks de l'activité Devoir. Ce dernier était soit proposé sous forme écrite via le feedback par commentaires, soit sous forme orale via le feedback PoodLL, soit les deux lorsque nous voulions expliquer une erreur de langue.

Nous avons demandé aux étudiants s'ils préféraient les commentaires personnalisés sous forme orale ou écrite. Ils ont répondu que les deux leur convenaient, tout en précisant qu'ils avaient chacun « leurs avantages » ou que les alterner était «l'idéal ». Lorsque nous avons donné des feedbacks oraux, nous avons veillé à avoir un ton enthousiaste, encourageant et bienveillant. En effet, si un étudiant n'avait pas fait toutes les activités de la séance, nous essayions d'abord de comprendre pourquoi il n'avait pas pu terminer («As-tu eu un problème technique? Ou as-tu juste manqué de temps?»). Nous avons également voulu lui donner des suggestions d'organisation (« Tu n'es pas obligé de faire toutes les activités en une seule fois, mais tu peux répartir ton travail sur plusieurs jours ») et des rappels sur l'importance de suivre assidûment ce cours de langue malgré, peut-être, sa « moindre » importance par rapport aux cours de spécialité ( Ce cours peut t'aider à progresser en français et donc mieux comprendre et réussir dans les autres cours! »).

nous avons voulu encourager les étudiants à travailler régulièrement ( $\mathrm{Je}$ te conseille de terminer les activités de la séance pour avancer ton travail à rendre à la fin du module petit à petit»). Ces conseils ont également été donnés sous forme écrite, accompagnés d'émoticônes pour «adoucir» les propos (Quintin et Masperi 10), ou juste pour égayer le message. Ces messages sortaient du cadre formel $\mathrm{du}$ courriel et de ses codes bien définis. Lorsque les étudiants ont posté des messages sur les forums « Pour le plaisir ! », nous nous sommes efforcée d'y répondre par un émoticône qui dit «J'aime ! » pour leur signaler que nous avons lu et apprécié leur message, comme illustré par la figure 1. Ces interventions peuvent sembler peu signifiantes, mais elles semblent propres à favoriser le développement d'une relation de confiance avec les étudiants. 


\section{JE SUIS DEVENUE UN GLACON}

par

dimanche 23 octobre 2016, 18:49

Bonjour,

En Grece quand il fait froid le monde dit une phrase tres frequente : "je suis devenue un glacon! "Mais en fait,on tous exagere car le "froid" en Grece est une situation tres rare,quelque chose que fait cette phrase drole en meme temps! :D

Modifier Supprimer Répondre

Re: JE SUIS DEVENUE Un GLACON

par Chae-Yeon Bournel-Bosson, lundi 31 octobre 2016, 13:31

Haha c'est mignon comme expression !

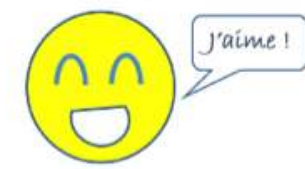

Figure 1. Interaction entre l'enseignant-tuteur et un étudiant dans le forum « Pour le plaisir! » de la séance 4.

Il est à noter enfin que nous avons inséré un enregistrement PoodLL en début de chaque séance, dans lequel nous présentons le déroulement et l'objectif principal de celle-ci. Cet enregistrement se termine par un mot d'encouragement. Le fait d'entendre la voix du tuteur semble rassurer et rappeler la présence humaine. Nous pouvons témoigner de l'importance de cette dimension à travers la remarque d'un.e étudiant.e : à la question «Auriez-vous préféré un petit texte introductif (au lieu d'un enregistrement) ? Si oui ou non, pourquoi ?», il/elle répond

«non [je n'aurais pas préféré un texte introductif à la place d'un enregistrement oral], parce que nous n'avons aucun contact avec le professeur avec cette plateforme, donc le enregistrement m'assure qu'il a une personne derrière cette technologie :-).» (Témoignage d'un.e étudiant.e)

\section{Conclusion}

Dans cette contribution, nous avons tenté de montrer que la mise en confiance de l'étudiant dans le cadre du distanciel peut être développée à travers quatre actions. L'étape préliminaire consiste en une maitrise technique de l'environnement d'apprentissage. Dans un deuxième temps, nous avons montré l'importance de partir des savoirs de l'étudiant pour scénariser une progression pédagogique dans un esprit réflexif. Nous avons également mis en évidence l'importance de renforcer le lien social entre les pairs, favorisant ainsi la formation d'une communauté d'apprentissage. Enfin, nous avons exploré quelques pistes pour renforcer l'interaction entre le tuteur et les étudiants : une communication régulière, bienveillante et appropriée dans ce contexte s'avère indispensable. Ces actions nous semblent fondamentales pour la mise en confiance, si ce n'est pour l'épanouissement, de l'étudiant dans un enseignement dispensé entièrement à distance, malgré les difficultés des étudiants à former une véritable communauté à distance en s'appropriant les espaces qui leurs sont propres (chat et forum). 
21 Nous pouvons étayer cette mise en confiance par le retour d'un.e étudiant.e à la fin de la formation (il s'agit d'un exemple parmi d'autres) :

«Je félicite la professeure [...] de sa bonne volonté à chaque réponse quand on a une doute, pour les correction toujours claires sur le point qu'on doit améliorer mais au même temps, exprimé d'une façon positive et que ne sont pas du tout démotivante. Merci :-) Bon courage pour vos prochain élèves ». (Témoignage d'un.e étudiant.e)

Ce cours est actuellement proposé à l'Université Paris-Diderot, et nous pouvons déjà voir avec les derniers retours des étudiants, mais aussi des tuteurs reprenant la formation, les améliorations à apporter, tant au niveau technique que pédagogique. Parlons FOU ! (B2) est également accessible à tous les enseignants de l'USPC qui souhaitent l'exploiter. Cela donnera certainement lieu à d'autres études qui permettront de nourrir la réflexion sur la notion de confiance dans le cadre de l'enseignement à distance.

\section{BIBLIOGRAPHIE}

Albernhe-Giordan, Huguette, et Chantal Charnet. Quand les enseignants rencontrent le numérique : innovation imposée ou attendue? Le cas de l'ENT dans le déploiement de l'Université numérique en Région Languedoc-Roussillon. 2005, Paris, France. 2005, http://sif2005.mshparisnord.org/pdf/ Albernhe.pdf. Dernier accès le 15 janvier 2017.

Bournel-Bosson, Chae-Yeon. Parlons FOU ! (niveau B2), perl.uspc.fr. Dernier accès le 10 février 2017. Castellotti, Véronique. « Diversité(s), histoire(s), compréhension...Vers des perspectives relationnelles et alterdidactiques pour l'appropriation des langues. » Recherches en didactique des langues et des cultures, $\mathrm{n}^{\circ} 12-1,2015$, rdlc.revues.org/420. DOI : 10.4000/rdlc.420.

Conseil de l'Europe. Un cadre européen commun de référence pour les langues : apprendre, enseigner, évaluer. Didier, 2010.

Dussarps, Clément. «L'abandon en formation à distance : Analyse socioaffective et motivationnelle. » Distances et Médiations des Savoirs, CNED-Centre national d'enseignement à distance, 2015, Communication éducative instrumentée : dispositifs médiatisés et leurs acteurs, dms.revues.org/1039. Dernier accès le 15 janvier 2017.

Dussarps, Clément, et Didier Paquelin, « Pratiques sociales en formation à distance. » Netcom, vol. 28, n³/4, 2014. DOI : 10.4000/netcom.1815.

Guillemet, Patrick. « Les étudiants préfèrent Facebook. » Distances et médiations des savoirs, $\mathrm{n}^{\circ} 6$, 2014, dms.revues.org/762. DOI : 10.4000/dms.762. Dernier accès le 15 janvier 2017.

Quintin, Jean-Jacques, et Monica Masperi. «Reliance, liance et alliance : opérationnalité des concepts dans l'analyse du climat socio-relationnel de groupes restreints d'apprentissage en ligne. » Alsic, vol. 13, 2010. DOI : 10.4000/alsic.1702. Dernier accès le 15 janvier 2017.

Servan Schreiber, Florence. «Le pouvoir de la gratitude. » Conférence TEDx, Paris, 2012, youtu.be/ nZUfJpVxUNI. Dernier accès le 01 février 2017. 
ANNEXES

\section{Annexe 1 - Questionnaire autoréflexif en séance 1}

\section{Quel genre d'étudiant êtes-vous ?}

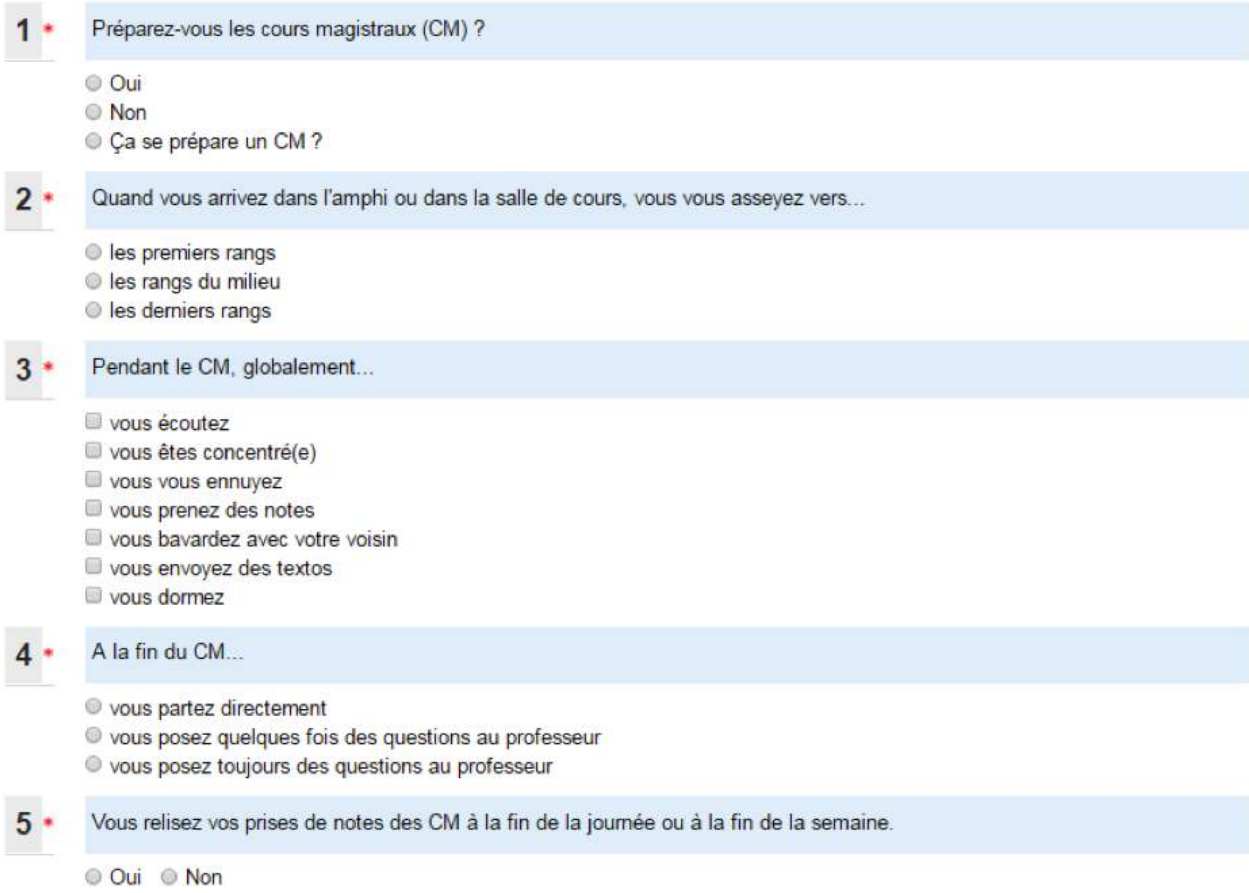




\section{Annexe 2 - Questionnaire autoréflexif en séance 4}

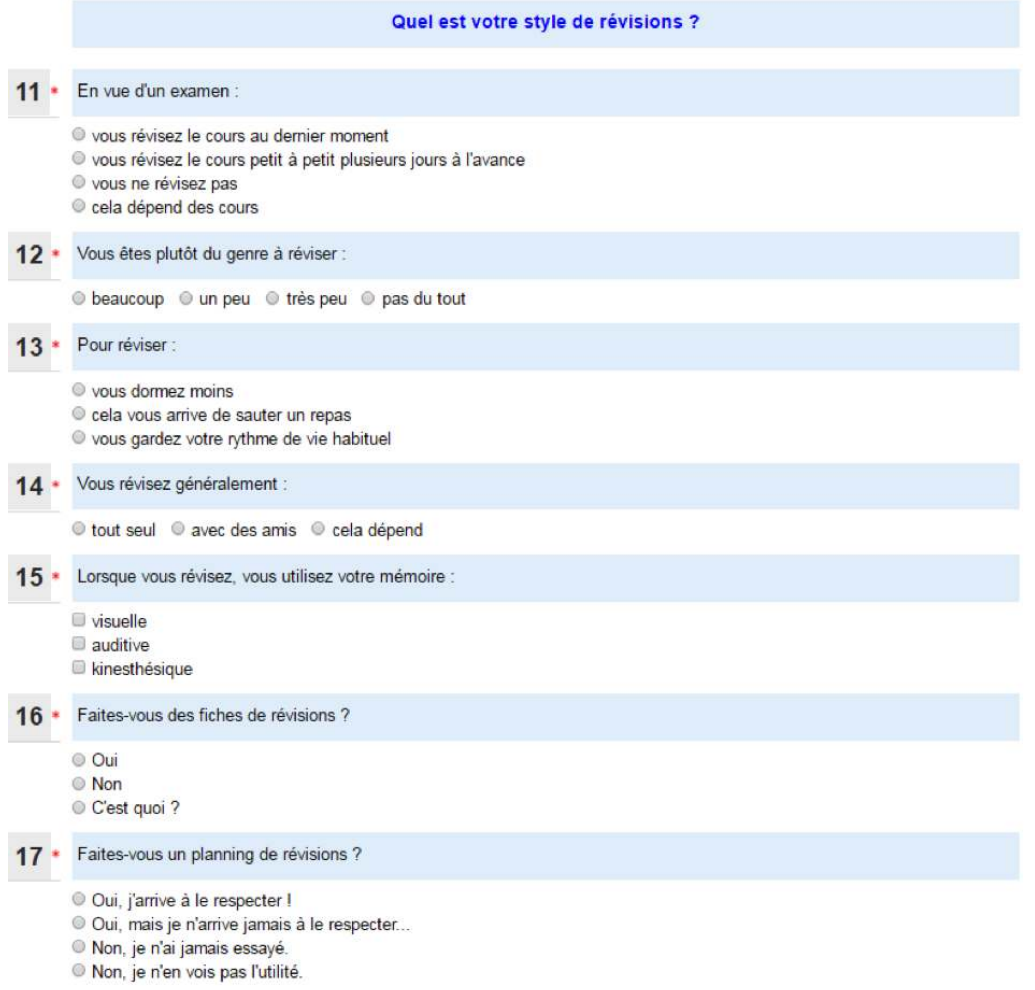

\section{NOTES}

1. http://perl.uspc.fr. Dernier accès le 10 février 2017.

2. La conception pédagogique de ce cours et le tutorat expérimental ont été assurés par ChaeYeon Bournel-Bosson.

3. Le niveau des étudiants a été évalué grâce au test de positionnement TCF de TV5 Monde. Depuis juin 2017, le niveau de langue des étudiants est évalué par un test de positionnement conçu par le Perl.

4. Commentaire d'un.e étudiant.e sur le cours Parlons FOU ! (B2) (questionnaire inclus dans le cours, disponible sur http://perl.uspc.fr). Dernier accès le 10 février 2017). Les commentaires sont reproduits fidèlement et contiennent les erreurs de langue des étudiants

5. PoodLL est un module d'enregistrement audio et vidéo.

\section{RÉSUMÉS}

Dans cette contribution, nous présentons les premiers retours d'expérience sur une formation de niveau B2 en français sur objectifs universitaires (FOU) entièrement dispensée à distance, intitulée Parlons FOU! (B2). Cette formation a été élaborée par le pôle d'élaboration de ressources 
linguistiques (Perl) - service partagé de l'université Sorbonne Paris Cité (USPC). Notre but a été de proposer des solutions en vue d'améliorer la confiance des étudiants dans une situation d'apprentissage via la plateforme pédagogique Moodle. Pour ce faire, nous avons conçu un parcours spécifique qui vise à développer la maîtrise technique de l'interface, à inciter les étudiants à réemployer leur savoir dans une visée réflexive, à créer et à renforcer le lien social entre eux, et à renforcer l'interaction entre l'enseignant-tuteur et les pairs.

In this paper, we present the first feedback on a distant learning course in French for Academic Purposes level B2, entitled Parlons FOU! (B2). This course was developed by the Linguistic Resource Development Unit (Perl), a common unit of the Université Sorbonne Paris Cité (USPC) consortium. Our goal was to find several solutions in order to improve the students' selfconfidence on a Moodle platform, a distant learning environment. To that purpose, we have designed a specific course in order to ensure students' technical expertise to use the interface, to encourage students to make the most of the skills acquired, to create and strengthen the social bond between them, and to improve the interaction between the teacher-tutor and the students themselves.

\section{INDEX}

Mots-clés : apprentissage des langues, confiance, enseignement à distance, français sur objectif universitaire (FOU), plateforme numérique d'apprentissage

Keywords : confidence, e-learning, French for academic purposes, language learning, learning management system (LMS)

\section{AUTEURS}

\section{CHAE-YEON BOURNEL-BOSSON}

Chae-Yeon Bournel-Bosson est maître de langue et conceptrice en FLE à l'université Paris-Diderot pour le pôle d'élaboration de ressources linguistiques (Perl) - service partagé de l'université Paris Cité Sorbonne. Elle est titulaire d'un master en linguistique appliquée à la didactique du FLE. Elle est également professeur de langues auto-entrepreneur.

cykim92@gmail.com

\section{JOVAN KOSTOV}

Jovan Kostov est ingénieur de recherche au pôle d'élaboration de ressources linguistiques (Perl) service partagé de l'université Paris Cité Sorbonne, après avoir été ATER (Paris-Sorbonne) et maître de langue (INALCO). En 2013, il a soutenu une thèse de doctorat en TAL appliqué à la didactique des langues. Il est membre de l'équipe d'accueil 4514 - PLIDAM - Institut national de langues et civilisations orientales (INALCO).

jovan.kostov@gmail.com 